\title{
CAPÍTULO 9: USO DA MEDICINA TRADICIONAL CHINESA NO TRATAMENTO PARA PACIENTES COM DISFUNÇÕES DA ARTICULAÇÃO TEMPOROMANDIBULAR: UM RELATO DE EXPERIÊNCIA
}

\author{
CAPÍTULO 9: USO DE MEDICINA TRADICIONAL CHINA EN EL \\ TRATAMIENTO DE PACIENTES CON DISFUNCIONES DE LA ARTICULACIÓN \\ TEMPOROMANDIBULAR: INFORME DE EXPERIENCIA
}

\section{CHAPTER 9: USE OF TRADITIONAL CHINESE MEDICINE IN TREATMENT FOR PATIENTS WITH TEMPOROMANDIBULAR JOINT DYSFUNCTIONS: AN EXPERIENCE REPORT}

\begin{abstract}
Camilla Siqueira de Aguiar ${ }^{1}$; Lohana Maylane Aquino Correia de Lima ${ }^{2}$; José Leonardo de Paiva e Sousa ${ }^{3}$ Milena Mello Varela Ayres de Melo ${ }^{4}$; Ricardo Eugenio Varela Ayres de Melo ${ }^{5}$
\end{abstract}

DOI: $\underline{\text { https://doi.org/10.31692/978-65-88970-04-1.81-85 }}$

\section{INTRODUÇ̃̃̃O}

A disfunção temporomandibular (DTM) foi descrita pela primeira vez em 1934 por James Costen, em que este defendia que mudanças nas condições dentárias provocavam sintomas otológicos (GIL, ZOTELLI, DE SOUZA, 2017). É considerada uma subclassificação das patologias musculoesqueléticas, apresentando uma flutuação dos seus sinais e sintomas ao longo do tempo (ZOTELLI, MEIRELLES, DE SOUZA, 2017). É uma síndrome associada a disfunções e anormalidades dos músculos da mastigação, ATM e estruturas associadas com a cabeça e cervical (COSTA et al., 2017). Esta disfunção é uma das principais causas de dor orofacial, de causa não dentária (MAGRO et al., 2016). Pode ser classificada em dois subgrupos: de origem articular, ou seja, disfunção em que os sinais e sintomas se relacionam com a articulação; e de origem muscular, em que os sinais e sintomas se relacionam com a musculatura do sistema estomatognático (JANUZZI et al., 2017).

Ela tem origem multifatorial e está relacionada com fatores articulares, neuromusculares, oclusais, como perda dentária, desgaste dos dentes, cáries, próteses mal adaptadas ou restaurações inadequadas, psicológicos, em que devido à tensão existente provoca um aumento da atividade muscular, o que gera fadiga e/ou espasmos, hábitos parafuncionais, como bruxismo e lesões traumáticas ou degenerativas da ATM (SILVA et al., 2017). A DTM é uma patologia

\footnotetext{
${ }^{1}$ Mestranda do Curso de Odontologia, Universidade Federal de Pernambuco, camilla.aguiar@

${ }^{2}$ Acadêmica do curso de Odontologia, Universidade Federal de Pernambuco, lohanawatso

${ }^{3}$ Fisioterapeuta e Doutor em Medicina Chinesa, Universidade Aberta do terapeuta, 1

${ }^{4}$ Acadêmico do curso de Medicina, Faculdade de Medicina de Olinda, milena varela

${ }^{5}$ Professor do curso de Odontologia, Universidade Federal de Pernambuco, revamelo@yahoo.
} 
que envolve problemas clínicos articulares e musculares na área orofacial, sendo caracterizada principalmente por dor muscular com pontos de gatilho, ou não, dores na mandíbula, região temporal, área pré-auricular e ouvido. Vários tipos de tratamentos são empregados com sucesso em DTMs, entretanto, com o aumento da busca por tratamentos odontológicos diferenciados e alternativos, tem ampliado a aplicação da acupuntura na Odontologia (PORPORATTI et al., 2015).

A acupuntura é uma técnica terapêutica da Medicina Tradicional Chinesa. Os efeitos da acupuntura são: diminuição da dor e o relaxamento muscular pela utilização de agulhas (COSTA et al., 2017). A acupuntura é uma terapia milenar, parte da Medicina Tradicional Chinesa, com mecanismos de ação energéticos e com propriedades analgésicas, antiinflamatórias, ansiolíticas, miorrelaxantes e ativadoras da função imunológica, é uma terapia efetiva, que traz como principais benefícios o relaxamento e diminuição da dor muscular em pacientes com DTMs (MAGRO et al., 2016). A auriculoterapia é uma alternativa de tratamento, pois a orelha é um microssistema do corpo e representa pontos específicos para tratar a dor no pavilhão auricular. Essa técnica estimula pontos específicos e individuais de cada paciente, além de ser de fácil manuseio, baixo custo e confortável para o paciente (PORPORATTI et al., 2015).

Essa técnica tem sido utilizada para amenizar e até mesmo sanar estados dolorosos, e sua principal indicação e utilização são em pacientes com DTM, apresentando resultados favoráveis, especialmente em relação à dor de origem muscular, mas também para casos de bruxismo; analgesia; trismo; controle prévio de ansiedade e estresse; medo ao tratamento odontológico; entre outros. Desse modo, realizada de maneira correta e seguindo as recomendações, a acupuntura além de favorecer a saúde, bem-estar e melhorar a qualidade de vida do paciente, tende a aperfeiçoar o tempo de trabalho no consultório odontológico (SILVA et al., 2017).

\section{RELATO DE EXPERIÊNCIA}

O Ambulatório de Cirurgia e Traumatologia Buco Maxilo Facial Odontologia da Universidade Federal de Pernambuco já atua há aproximadamente no que se refere a prevenção, diagnóstico, tratamento e controle a pacientes, na sua maioria carentes e principalmente do estado de Pernambuco. Junto com a PROEXC, como projeto de extensão, a ação do projeto está voltada para a área da saúde, onde visa a melhor qualidade de 
vida das pessoas que apresentam traumas de faces ou patologias bucais, em uma atividade que vai desde o acolhimento até a sua total cura.

A disfunção da articulação temporomandibular é a patologia mais frequente entre os pacientes atendidos no Ambulatório de Cirurgia e Traumatologia Buco Maxilo Facial. Sintomas como enxaquecas, dores e zumbidos nos ouvidos, limitação da abertura bucal, luxação da articulação, entre outros, são frequentes nesses pacientes que em consequência alteram a sua qualidade de vida, relatando dificuldades para exercer funções do cotidiano, como trabalhar, dormir, mastigar, falar entre outros. Considerando a necessidade do serviço e aspirando um melhor resultado menos invasivo e a curto prazo, esse projeto visa associar os tratamentos convencionas das disfunções da articulação temporomandibular com as técnicas da Medicina Tradicional Chinesa.

Com o enfoque em DTM, os atendimentos ocorrem através da aplicação da terapêutica por profissionais qualificados e observação do manejo por parte da equipe de extensão com o principal objetivo sendo de fornecer um tratamento para os pacientes que apresentam disfunções da articulação temporomandibular, multidisciplinar que une a Cirurgia Buco Maxilo Facial com as técnicas da Medicina Tradicional Chinesa, além de fornecer aos alunos do projeto a vivência da aplicação da Medicina Tradicional Chinesa nos tratamentos das disfunções da articulação temporomandibular.

O projeto de extensão acontece 01 (uma) vez na semana, totalizando 4 encontros mensais. As atividades são realizadas nas quartas-feiras a partir das 16:00 horas até às 20:00, totalizando 04 (quatro) horas semanais. As atividades ocorrem no Ambulatório de Cirurgia e Traumatologia Buco Maxilo Facial da UFPE e o público-alvo está relacionado com os pacientes que são usuários do serviço.

A relação ensino, pesquisa e extensão desse projeto se caracteriza pela presença dos acadêmicos, que são de grande valia para o ensino e prática supervisionada pelo docente, oferecendo ao aluno um maior aprendizado e possibilidade de executar procedimentos na áre $\mathrm{O}$ atendimento à população, destacando-se a população carente, utilizando o método de registros qualificados e quantificados implicando na realização de pesquisas, que maioria casos clínicos para que outros profissionais tenham acesso à metodologia e técnica de cada caso e como resultado formal frente a esta Instituição de Ensino Superior. Possibilita que os alunos atendam uma demanda de pacientes nos mais diversos problemas da articulação temporomandibular proporcionando ao mesmo um conhecimento maior do que exigido na grade curricular do curso. 
Além disso, há a emissão de certificados, que, em um futuro próximo, o acadêmico poderá inserir em seu curriculum, frente a concursos, residências odontológicas e multiprofissionais, levando o aprendizado e transferindo como formador de opinião. Podemos observar também, que esses tratamentos alternativos, baseados na Medicina Tradicional Chinesa por muitas vezes são desconhecidos como forma de tratamento pelos alunos dos cursos de graduação em saúde e o projeto de extensão vem agregar ao currículo do estagiário esse conhecimento sobre essas diversas formas de tratamentos complementares, permitindo assim uma melhor condução dos seus casos e despertar curiosidade e interesse sobre todas as opções de manejo terapêutico possíveis, nesses futuros profissionais

\section{CONCLUSÕES}

Observa-se com esse projeto de extensão que a inclusão do uso da Medicina Tradicional Chinesa no manejo terapêutico das disfunções da articulação temporomandibular e a possibilidade de vivência pelos estagiários do serviço a sequência completa do início ao final das necessidades interventivas no processo ensino/aprendizagem das técnicas utilizadas.

\section{REFERÊNCIAS}

COSTA, Deborah Santos Morgado et al. A UTILIZAÇÃO DA ACUPUNTURA EM SINAIS E SINTOMAS DAS DISFUNÇÕES TEMPOROMANDIBULARES: REVISÃO SISTEMÁTICA. Revista Univap, v. 22, n. 40, p. 667, 2017.

GIL, Maria Lúcia Bressiani; ZOTELLI, Vera Lúcia Rasera; DE SOUSA, Maria da Luz Rosário. Acupuntura como alternativa para eltratamiento de ladisfunción temporomandibular. Revista

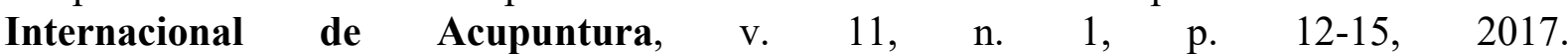

JANUZZI, Marcella Santos et al. Terapia complementar através da acupuntura para dor orofacial. ARCHIVES OF HEALTH INVESTIGATION, v. 6, 2017.

MAGRO, Katia O. et al. Acupuntura: tratamento alternativo nas dores orofaciais. UnidorUnidade de Dor e Deformidade Orofacial, 2016.

PORPORATTI, André Luís et al. Acupuncturetherapeuticprotocols for the management of temporomandibular disorders. Revista Dor, v. 16, n. 1, p. 53-59, 2015.

SILVA, PatriseThomasi da et al. Acupuntura no tratamento das temporomandibulares musculares. 2017.

ZOTELLI, Vera Lucia Rasera; MEIRELLES, Maria Paula Maciel Rando; DE SOUSA, Maria da Luz Rosário. Uso da acupuntura no manejo da dor em pacientes com alterações na articulação temporomandibular (ATM). Revista de Odontologia da Universidade 
Cidade de São Paulo, v. 22, n. 2, p.185-188, 2017. 